\title{
Structural analysis of human Cdc20 supports multisite degron recognition by $\mathrm{APC} / \mathrm{C}$
}

\author{
Wei Tian ${ }^{a, 1}$, Bing Li ${ }^{a, b, 1}$, Ross Warrington ${ }^{a, b}$, Diana R. Tomchick ${ }^{c}$, Hongtao $\mathrm{Yu}^{\mathrm{a}, \mathrm{b}, 2}$, and Xuelian Luo ${ }^{\mathrm{a}, 2}$ \\ aDepartment of Pharmacology, ${ }^{b}$ Howard Hughes Medical Institute, and 'Department of Biophysics, University of Texas Southwestern Medical Center, Dallas, \\ TX 75390
}

Edited by Stephen C. Harrison, Howard Hughes Medical Institute and Children's Hospital, Harvard Medical School, Boston, MA, and approved September 24, 2012 (received for review August 3, 2012)

The anaphase-promoting complex/cyclosome (APC/C) promotes anaphase onset and mitotic exit through ubiquitinating securin and cyclin B1. The mitotic APC/C activator, the cell division cycle 20 (Cdc20) protein, directly interacts with APC/C degrons-the destruction (D) and KEN boxes. APC/C $C^{\mathrm{Cdc20}}$ is the target of the spindle checkpoint. Checkpoint inhibition of $A P C / C^{\mathrm{Cdc} 20}$ requires the binding of a BubR1 KEN box to Cdc20. How APC/C recognizes substrates is not understood. We report the crystal structures of human Cdc20 alone or bound to a BubR1 KEN box. Cdc20 has a disordered $\mathrm{N}$-terminal region and a $\mathrm{C}$-terminal WD40 $\beta$ propeller with a preformed KEN-box-binding site at its top face. We identify a second conserved surface at the side of the Cdc20 $\beta$ propeller as a D-box-binding site. The D box of securin, but not its KEN box, is critical for securin ubiquitination by $A P C / C^{C d c 20}$. Although both motifs contribute to securin ubiquitination by $A P C / C^{\mathrm{Cdh} 1}$, securin mutants lacking either motif are efficiently ubiquitinated. Furthermore, D-box peptides diminish the ubiquitination of KEN-box substrates by APC/C $C^{\mathrm{Cdh} 1}$, suggesting possible competition between the two motifs. Our results indicate the lack of strong positive cooperativity between the two degrons of securin. We propose that low-cooperativity, multisite target recognition enables APC/C to robustly ubiquitinate diverse substrates and helps to drive cell cycle oscillations.

mitosis | crystallography | multivalency | molecular recognition

$\mathbf{T}^{\mathrm{h}}$ he anaphase-promoting complex/cyclosome $(\mathrm{APC} / \mathrm{C})$ is a multisubunit ubiquitin ligase that mediates the ubiquitination of a multitude of substrates to drive cell cycle progression (1-3). At the metaphase-anaphase transition, $\mathrm{APC} / \mathrm{C}$ in complex with its mitotic activator $\mathrm{Cdc} 20$ (APC/C $\mathrm{C}^{\mathrm{Cd} 20}$ ) ubiquitinates securin and cyclin $\mathrm{B} 1$, triggering their destruction to allow separase activation and cohesin cleavage. In anaphase, APC/C bound to the $\mathrm{Cdc} 20$ homolog Cdh1 (APC/C $\mathrm{C}^{\mathrm{Cdh} 1}$ ) further mediate the ubiquitination of cyclin $\mathrm{B} 1$ and other mitotic regulators, resulting in their degradation and mitotic exit.

$\mathrm{Cdc} 20$ and $\mathrm{Cdh} 1$ activate $\mathrm{APC} / \mathrm{C}$ by contributing to substrate recognition, among other mechanisms (1-3). All APC/C substrates contain one or more of several types of short peptide motifs with highly degenerate sequences, referred to as APC/C degrons. For example, cyclin B1 contains a destruction (D) box with the consensus of $\mathrm{RXXLX}_{4-5} \mathrm{~N}$ (X, any residue), whereas securin contains both a D box and a KEN box (4-6). Biochemical and biophysical studies have established that Cdh1 and the APC/C subunit Apc10 serve as coreceptors for the D box, with the D box bridging an interaction between the two (7-11).

APC/C $C^{\mathrm{Cdc} 20}$ is the molecular target of the spindle checkpoint, which prevents premature sister-chromatid separation and aneuploidy $(3,12-15)$. In response to kinetochores not properly attached to the spindle microtubules, the spindle checkpoint inhibits $\mathrm{APC} / \mathrm{C}^{\mathrm{Cdc} 20}$ using multiple mechanisms. Among these mechanisms, the mitotic checkpoint complex (MCC; consisting of BubR1/Mad3, Bub3, Mad2, and Cdc20) anchors Cdc20 at a site on $\mathrm{APC} / \mathrm{C}$ that is different from the site bound by free Cdc20, presumably blocking the productive engagement of the $\mathrm{D}$ box with Cdc20 and Apc10 (16-21). BubR1/Mad3 contains two KEN boxes (KEN1 and KEN2), both of which are required for the spindle checkpoint (22-25). BubR1/Mad3 has thus been suggested to inhibit APC/C by acting as a pseudosubstrate, with its KEN boxes competing against those of the substrates for Cdc20 binding $(22,23)$.

To better understand how APC/C recognizes its substrates, we determined the crystal structures of human Cdc20 alone or bound to the KEN1 box of BubR1, revealing a preexisting KENbox-binding site at the top of the WD40 $\beta$ propeller. Structurebased mutagenesis then defined a second conserved surface on the side of the $\beta$ propeller as a D-box-contacting site. Surprisingly, despite binding to distinct sites on Cdc20, the KEN and D boxes of securin do not apparently interact with $\mathrm{APC} / \mathrm{C}$ in a highly cooperative manner. The KEN box is largely dispensable for securin ubiquitination by $\mathrm{APC} / \mathrm{C}^{\mathrm{Cdc} 20}$. This lack of strong cooperativity between the two degrons also applies to APC/ $\mathrm{C}^{\mathrm{Cdn} 1}$, but the KEN and D boxes have comparable contributions in this case. D-box peptides inhibit APC/C-mediated ubiquitination of KEN-box substrates, suggesting competition between the two motifs. Our results indicate that the multiple APC/C degrons of securin do not engage $\mathrm{APC} / \mathrm{C}$ in a highly cooperative manner. A single functional APC/C degron within a substrate supports APC/C $\mathrm{C}^{\mathrm{Cdh} 1}$-dependent ubiquitination. We propose that this low-cooperativity, multisite substrate recognition mechanism expands the substrate spectrum of $\mathrm{APC} / \mathrm{C}$, allowing it to attenuate the cellular concentrations of myriad substrates with one or more functional degrons.

\section{Results and Discussion}

Structures of Human Cdc20 by Itself or in Complex with BubR1 KEN1. To determine the structure of human Cdc20, we expressed and purified from Sf9 insect cells a Cdc20 truncation mutant with its N-terminal 60 residues deleted $\left(\mathrm{Cdc} 20^{\Delta \mathrm{N} 60}\right)$. Cdc $20^{\Delta \mathrm{N} 60}$ contained all known functional motifs of $\mathrm{Cdc} 20$ (3), including the $\mathrm{C}$ box, the KEN box, and the Mad2-interacting motif (MIM), and actively supported APC/C- and D-box-dependent ubiquitination of cyclin B1 (Fig. $1 A$ and Fig. S1). We obtained crystals of Cdc $20^{\Delta \mathrm{N} 60}$ that diffracted to about $3 \AA$, but could not determine its structure using molecular replacement. We thus made additional Cdc20 truncation mutants $\mathrm{Cdc} 20^{\Delta \mathrm{N} 70}$ and $\mathrm{Cdc} 20^{\Delta \mathrm{N} 80}$, obtained crystals of $\mathrm{Cdc} 20^{\triangle \mathrm{N} 80}$ that diffracted to $2.0 \AA$, and determined its structure using molecular replacement with the coordinates of Wdr5 as the search model (Table S1). Only the WD40 domain of Cdc20 $0^{\Delta \mathrm{N} 80}$ was well defined in the structure. Both the N-terminal region (residues 81-164) and the C-terminal region (residues 477-499; including the IR motif) of $\mathrm{Cdc} 20^{\Delta \mathrm{N} 80}$ were disordered and not visible in the structure. We then obtained an unrefined structure model of $\operatorname{Cdc} 20^{\Delta \mathrm{N} 60}$ using molecular

Author contributions: W.T., B.L., R.W., and X.L. performed research; D.R.T., H.Y., and X.L. analyzed data; H.Y. and X.L. designed research; and H.Y. and X.L. wrote the paper.

The authors declare no conflict of interest.

This article is a PNAS Direct Submission.

Data deposition: The atomic coordinates have been deposited in the Protein Data Bank, www.pdb.org (PDB ID codes 4GGA, 4GGC, and 4GGD).

${ }^{1}$ W.T. and B.L. contributed equally to this work.

${ }^{2}$ To whom correspondence may be addressed. E-mail: xuelian.luo@utsouthwestern.edu or hongtao.yu@utsouthwestern.edu.

This article contains supporting information online at www.pnas.org/lookup/suppl/doi:10. 1073/pnas.1213438109/-/DCSupplemental. 
B

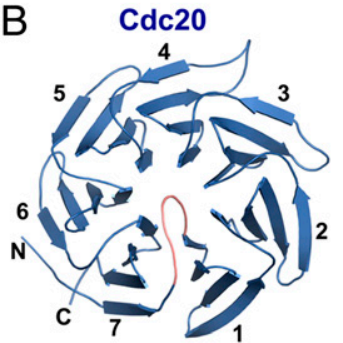

C

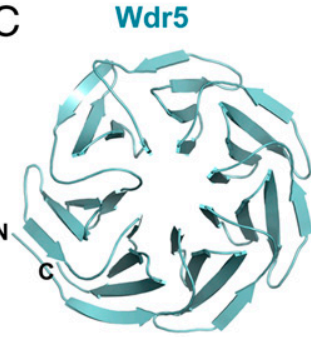

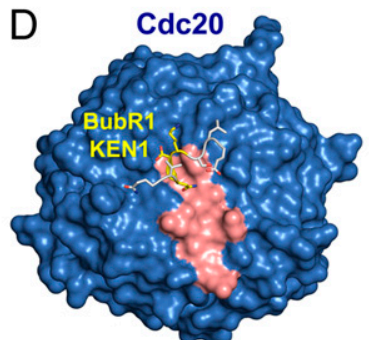

E

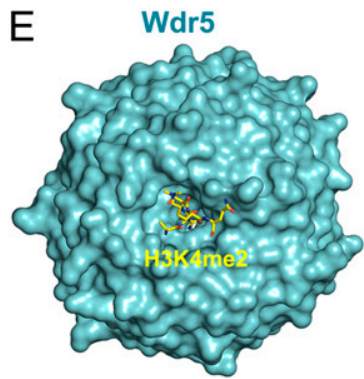

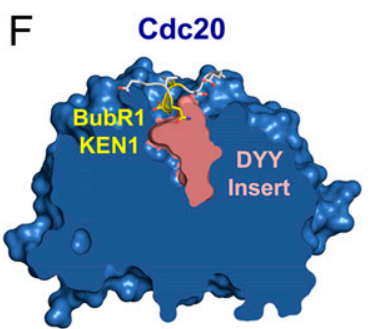

G

Wdr5

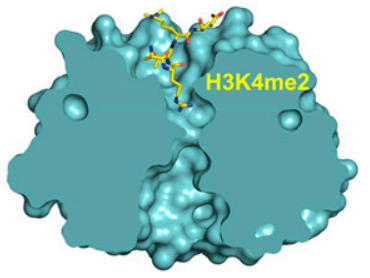

Fig. 1. Human $\mathrm{Cdc} 20$ has a preformed KEN-boxbinding site at the top face of its WD40 $\beta$ propeller. (A) Domains and motifs of human Cdc20. MIM Mad2 Interacting Motif. (B) Ribbon diagram of human Cdc20. The $\mathrm{N}$ and $\mathrm{C}$ termini and the seven blades of the WD40 $\beta$ propeller are labeled. The DYY insert in the loop connecting blades 1 and 7 is colored salmon. All structure figures in this report are generated with PyMOL (www.pymol.org). (C) Ribbon diagram of Wdr5 (PDB ID code 2GNQ). (D) Surface drawing of the $\mathrm{Cdc} 20^{\triangle N 70}$-BubR1 KEN1 complex with BubR1 KEN1 shown as sticks. The DYY insert is colored salmon. $(E)$ Surface drawing of the Wdr5-histone $\mathrm{H} 3$ complex (PDB ID code $2 \mathrm{H} 9 \mathrm{O}$ ), with the bound $\mathrm{H} 3$ peptide shown as sticks. $(F)$ A crosssectional view of the surface drawing of $\mathrm{Cdc} 20$ BubR1 KEN1 in $D$. (G) A cross-sectional view of the surface drawing of Wdr5-H3 in $E$.

replacement with $\mathrm{Cdc} 20^{\Delta \mathrm{N} 80}$ as the search model, but could not refine the structure due to poor quality diffraction data. Nonetheless, it was clear that the $\mathrm{N}$ - and C-terminal regions of the functional Cdc $20^{\Delta \mathrm{N} 60}$ (residues 61-164 and 477-499) were also disordered.

The WD40 domain of Cdc20 expectedly folds into a canonical seven-bladed $\beta$ propeller. Unlike Wdr5 and other WD40 domain structures (26), Cdc20 does not have a deep ligand-binding pocket at the top face of its $\beta$ propeller (Fig. $1 B-G$ ). Instead, an insert (containing residues 183-188) in the loop connecting the seventh and first blades of the Cdc20 propeller occupies this pocket (Fig. $1 B, D$, and $F$ ), forming numerous contacts with surrounding residues and creating a flat top surface.

To gain insight into how $\mathrm{Cdc} 20$ recognized $\mathrm{APC} / \mathrm{C}$ degrons, we determined the structure of $\operatorname{Cdc} 20^{\Delta \mathrm{N} 70}$ bound to the KEN1 box of human BubR1. The structures of free and KEN-bound Cdc20 were virtually identical, indicating that KEN-box binding did not induce conformational changes of Cdc20. Only eight residues of the KEN1 peptide (from E23 to Q30), including the KEN motif, were visible in the structure (Fig. 1D). The KEN motif (residues 26-28) folds into an irregular 3 helix that binds at the top face of the propeller. A highly conserved DYY motif of the aforementioned insert of Cdc20 establishes the base of the KEN-boxbinding site, contacting the KEN backbone and the side chains of E27 and N28 (Figs. $1 F$ and $2 A$ ). E27 and N28 make additional contacts with A357, T377, Q401, and R445 (Fig. 2A). The K26 side chain forms hydrogen bonds with the backbone carbonyl of N329 and the side chains of N329 and N331.

Because Cde20 has a KEN box in its N-terminal region, we had envisioned that Cdc20 might adopt an auto-inhibited conformation
A

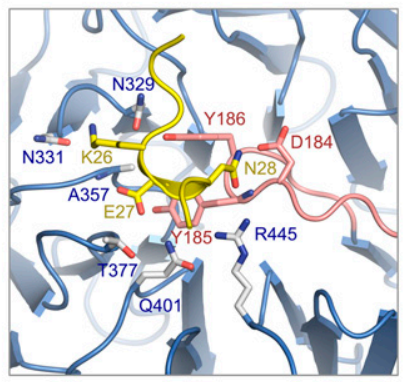

C

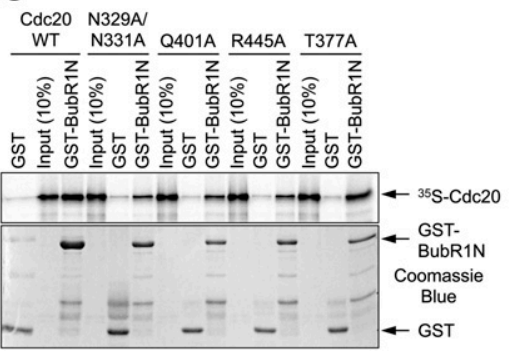

B
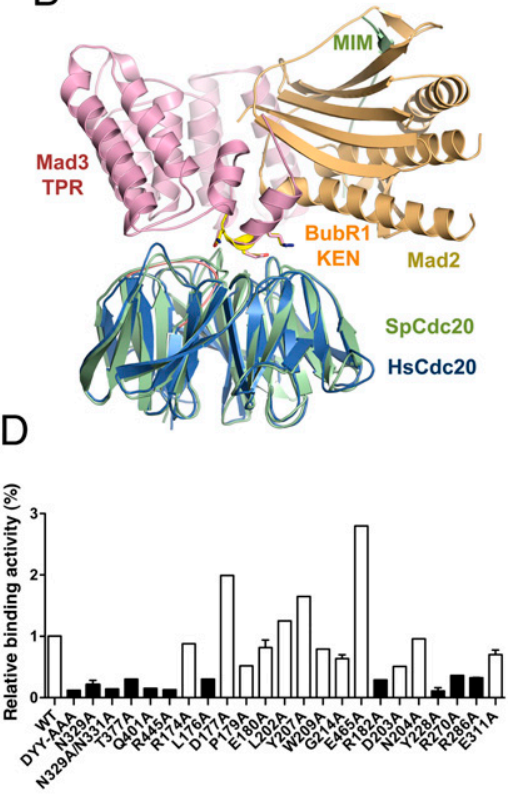

Fig. 2. Identification of critical KEN-box-binding residues of $\mathrm{Cdc20}$. (A) A close-up view of the interface between $\mathrm{Cdc} 20$ and BubR1 KEN1. The interacting residues are shown as sticks and labeled. The coloring scheme is the same as for Fig. 1. (B) Overlay of the ribbon diagrams of human $\mathrm{Cdc} 20$ BubR1 KEN1 (colored blue and yellow, respectively) and the fission yeast Cdc20-Mad3-Mad2 (PDB ID code 4AEZ; colored green, gold, and pink, respectively). (C) Binding between GST-BubR1N (residues 15-237) and the in vitro translated ${ }^{35}$ S-labeled indicated Cdc20 wild-type (WT) or mutant proteins. The phosphoimager image and Coomassie blue staining of the same gel are shown in Upper and Lower, respectively, with the positions of ${ }^{35} \mathrm{~S}-\mathrm{Cdc} 20, \mathrm{GST}$, and GST-BubR1N indicated by arrows. (D) Quantification of the binding between GST-BubR1N and Cdc20 WT and mutant proteins. Columns of Cdc20 mutants with less than $50 \%$ of the binding activity of Cdc20 WT are in black. 
with its KEN box binding to its WD40 domain intramolecularly to limit the access of KEN-box-containing substrates. However, our structure of the free Cdc20 did not support such a model. The $\mathrm{N}$ terminal region of Cdc20 was disordered. The KEN-box-binding site of free Cdc20 did not have unassigned electron density that might have belonged to the Cdc20 KEN box. Deletion of the KEN box of Cdc20 did not alter its ability to activate APC/C (Fig. S1 and see Fig. $4 A$ below)

Comparison of our structures with that of the fission yeast MCC revealed no gross conformational changes of Cdc20 upon binding to Mad2 and the N-terminal TPR domain of Mad3, except for the disordered-to-ordered transition of the Mad2interacting motif (MIM) of Cdc20 (Fig. 2B) (21). The interactions between human Cdc20 and BubR1 KEN1 were also highly similar to those observed between the fission yeast Cdc20 and Mad3 (Fig. 2B) (21), indicating that the mode of KEN box recognition by $\mathrm{Cdc} 20$ was evolutionarily conserved.

Mutagenesis Analysis of the KEN-Box-Binding Site of Cdc20. To validate the functional relevance of the observed interface between Cdc20 and BubR1 KEN1, we mutated the KEN-box-contacting residues of $\mathrm{Cdc} 20$ and tested the binding of these Cdc20 mutants to N-terminal fragments of BubR1 (residues 15-237, 20-237, and 15-210) containing the KEN1 box. Binding of BubR1N to Cdc20 was KEN-box-dependent, as a shorter BubR1N fragment (residues 30-210) lacking KEN1 did not bind Cdc20 efficiently (Fig. S2A). Mutations of KEN1-binding Cde20 residues, including the DYY motif, N329, N331, Q401, and R445, greatly reduced Cdc20 binding to BubR1N (Fig. $2 C$ and $D$ ). The T377A mutation had a weaker but still appreciable effect. These results validated the KEN-box-binding site of Cdc20.

Addition of the synthetic BubR1 KEN1 peptide reduced, but did not abolish, Cdc20 binding to BubR1N, indicating that other regions of BubR1N contributed to Cdc20 binding (Fig. S2B). Consistently, mutations of several conserved residues at the top face of the $\beta$ propeller, including R182 and Y228, also decreased Cdc20 binding to BubR1N (Fig. 2D). These Cdc20 residues might contact the TPR domain of BubR1N, as the corresponding fission yeast $\mathrm{Cdc} 20$ residues contacted the Mad3 TPR domain in the yeast MCC structure (21).

Defining the D-Box-Binding Site of Cdc20. Electron microscopy studies have implicated Cdh1 and Apc10 as the coreceptor for the $\mathrm{D}$ box $(10,11)$. It is highly likely that Cdc20 and Apc10 can also form a coreceptor for the D box. Despite repeated attempts, we could not obtain structures of $\mathrm{Cdc} 20$ bound to the D-box peptides of cyclin B1 or securin. In the absence of APC/C, Cdc20 alone might not bind to the D box with appreciable affinity. Indeed, we could not detect Cdc20 binding to beads that were covalently coupled to the cyclin B1 D box peptide. We also could not detect binding between human Apc10 and the D box peptide using either standard pulldown experiments or NMR spectroscopy. Addition of human Apc10 did not promote an interaction between Cdc20 and the D box peptide.

Inspection of the molecular surface of the Cdc20 WD40 domain revealed two conserved patches (Fig. $3 A$ ). The first patch at the top of the $\beta$ propeller is the KEN-box-binding site. A second conserved patch is located between blades 1 and 7 at the side of the $\beta$ propeller and is formed by residues R174, L176, D177, P179, L202, Y207, W209, and E465 (Fig. $3 A$ and $B$ ). Several Cdh1 residues corresponding to these Cdc20 residues were implicated in D-box binding in chemical crosslinking experiments (7). Furthermore, during the many attempts to cocrystalize Cdc20 and D-box peptides, we managed to determine the structure of the Cdc20 WD40 domain to very high resolution $(1.35 \AA)$. In this structure, an MPD (2-methyl-2,4-pentanediol) molecule was bound in a conspicuous hydrophobic pocket at the center of this patch (Fig. $3 A$ ), suggesting that this pocket might be a functional ligand-binding site. Indeed, in the crystal structure of the fission yeast MCC, a leucine side chain from a neighboring molecule inserted into this pocket in Cdc20 (21). Thus, this second conserved patch is likely involved in D-box binding, with the deep pocket accommodating the leucine in the RXXL consensus motif of the D box.

We next mutated residues in this patch and tested the ability of these Cdc20 mutants to promote cyclin B1 ubiquitination, which was critically dependent on the D box. All these mutations, except for W209A, significantly diminished the ability of Cdc20 to activate $\mathrm{APC} / \mathrm{C}$-mediated ubiquitination of cyclin B1 (Fig. $3 C$ ). As controls, mutations of the KEN-box-binding residues at the top face of the $\beta$ propeller, including DYY-AAA, T377A, Q401A, and R445A, had no effects. Furthermore, with the exception of L176A, all ubiquitination-deficient mutants affecting the putative D-box-binding residues still bound to BubR1N (Fig. 2D). In fact, for unknown reasons, D177A, Y207A, and E465A even bound to BubR1N more efficiently than the wild-type Cdc20 did. These mutants were thus correctly folded and presumably conferred weaker APC/C-stimulatory activity due to deficient D-box binding. If so, we would expect that these mutations should not further
A

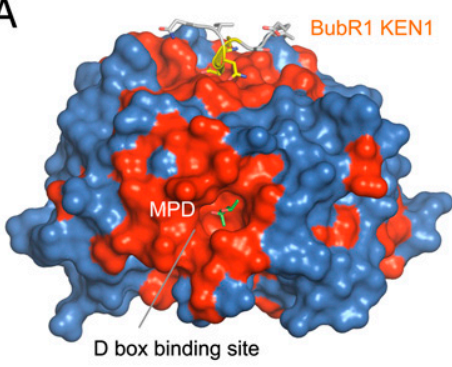

C

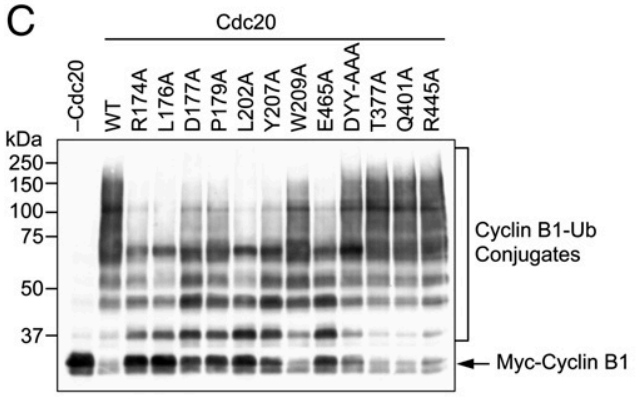

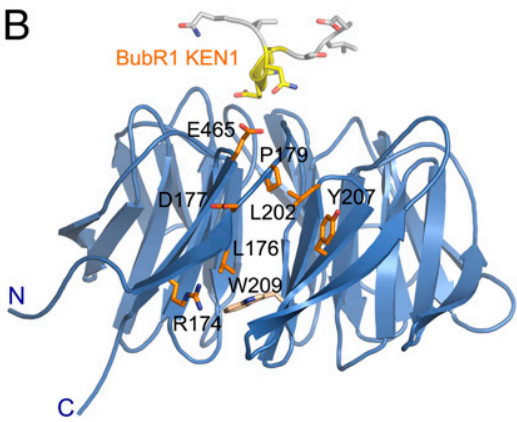

D Cyclin $\mathrm{B} 1 \Delta \mathrm{D}-\ldots+++$ Cyclin B1 WT +++-$\mathrm{Cdc} 20^{\triangle N 60} \mathrm{D} 177 \mathrm{~A}-\cdots+-++$ $\mathrm{Cdc} 2 \mathrm{O}^{\triangle \mathrm{N} 60}-+--+$ $\mathrm{APC} / \mathrm{C}+++++++$

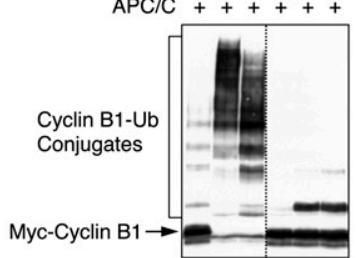

Fig. 3. Identification of a conserved D-box-binding site on the side of $\mathrm{Cdc} 20 \beta$ propeller. $(A)$ Surface diagram of $\mathrm{Cdc} 20^{\mathrm{WD} 40}$. The conserved $\mathrm{Cdc} 20$ residues are colored red. The BubR1 KEN1 peptide (shown as sticks with the KEN motif colored yellow) is grafted onto this structure to indicate the position of the KEN-box-binding site. The MPD molecule bound to a deep pocket is shown as green sticks. (B) Ribbon diagram of Cdc20-BubR1 KEN1 with BuBR1 KEN1 and the conserved Cdc20 residues at the D-boxbinding site shown as sticks. Residues whose mutations diminish APC/C activity toward cyclin B1 are shown in orange. W209 is shown in gray. (C) The indicated Cdc20 WT and mutant proteins were in vitro translated in rabbit reticulocyte lysate and assayed for their ability to activate the ubiquitination of human Myc-cyclin B1 (1-102) by Xenopus APC/C. The reaction mixtures were blotted with anti-Myc. The positions of unmodified cyclin B1 and cyclin B1-ubiquitin conjugates are labeled. $(D)$ Purified $C \mathrm{Cdc}_{20} \mathrm{DN}^{\mathrm{N} 60}$ and Cdc20 ${ }^{\triangle N 60}$ D177A were assayed for their ability to activate the ubiquitination of human Myc-cyclin $\mathrm{B} 1^{1-102}$ WT or $\Delta \mathrm{D}$ by Xenopus APC/C. The reaction mixtures were blotted with anti-Myc. The positions of unmodified cyclin B1 and cyclin B1-ubiquitin conjugates are labeled. 
diminish the basal ubiquitination of cyclin $\mathrm{B} 1$ with its $\mathrm{D}$ box mutated $(\Delta \mathrm{D})$. Indeed, although Cdc20 D177A ubiquitinated cyclin B1 less efficiently than the wild-type Cdc20 did (as evidenced by the lower molecular masses of the ubiquitin conjugates generated), Cdc20 WT and D177A weakly monoubiquitinated cyclin $\mathrm{B} 1 \Delta \mathrm{D}$ to a similar extent (Fig. $3 D$ ).

Taken together, these results suggest that this second conserved surface is involved in D-box binding. We further speculate that the deep hydrophobic pocket formed by L176, P179, L202, Y207, and W209 might accommodate the leucine in the RXXL motif, whereas D177 or E465 or both might form favorable electrostatic interactions with the arginine in that motif. Cdc20 has been implicated as a viable molecular target for the development of new anti-mitotic class of anticancer drugs, as its inactivation blocks mitotic exit and triggers apoptosis in human cancer cells (27). The presence of a deep pocket on the surface of Cdc20 suggests that it is indeed druggable. Our high-resolution structure of human Cdc20 will aid the design and development of specific Cdc20 inhibitors through virtual screens of compound libraries.

KEN Box of Securin Is Dispensable for Its Ubiquitination by APC/C Cdc20 $^{\text {. }}$ Our structural analysis of human Cdc20 presented above suggested that the two key APC/C degrons, the KEN and D boxes, bound at two different sites on Cdc20. This feature of Cdc20 appeared to be conserved in the fission yeast Cdc20 (21). Several APC/C substrates, including securin, contained both KEN and D boxes $(6,28)$. An attractive hypothesis was that the KEN and D boxes of securin could cooperatively engage $\mathrm{Cdc} 20$ in a bipartite interaction, thus enhancing its binding affinity and specificity toward Cdc20. However, the KEN box is largely dispensable for binding of securin to APC/ $\mathrm{C}^{\mathrm{Cdc} 20}$ and for $\mathrm{APC} / \mathrm{C}^{\mathrm{Cdc} 20}$-dependent ubiquitination of securin (Fig. S3) (29-31). These findings do not support highly cooperative bivalent recognition of the KEN and $\mathrm{D}$ boxes by APC/C $\mathrm{Cdc20}$.

One possibility was that although Cdc20 bound to the KEN box, such a binding was incapable of supporting ubiquitination by APC/C. We tested whether APC/C ${ }^{\mathrm{Cdc20}}$ could ubiquitinate the central fragment of Bub1 (residues 520-653), which contained two KEN boxes but lacked a D box $(32,33)$. Indeed, Cdc20 supported ubiquitination of Bub1 ${ }^{520-653}$, albeit at a much reduced efficiency compared with Cdh1 (Fig. 4A). Mutation of the KEN box of Cdc20 did not further enhance Bub1 ubiquitination
(Fig. 4A), again arguing against an autoinhibitory mechanism involving an intramolecular Cdc20 KEN-WD40 interaction.

We next tested whether the KEN box of securin could mediate ubiquitination in the absence of the $\mathrm{D}$ box. We created securin mutants with its $\mathrm{D}$ box mutated $(\mathrm{mD})$ or with both the $\mathrm{D}$ and KEN boxes mutated (mDK). Compared with securin WT, securin $\mathrm{mD}$ formed ubiquitin conjugates with lower molecular mass in the presence of $\mathrm{APC} / \mathrm{C}^{\mathrm{Cdc} 20}$, indicating a requirement for the $\mathrm{D}$ box in securin ubiquitination (Fig. $4 B$, compare lanes 2 and 10). Interestingly, although securin $\mathrm{mK}$ (with the KEN box mutated) was ubiquitinated as well as securin WT (compare lanes 2 and 6), securin $\mathrm{mDK}$ was less efficiently ubiquitinated compared with securin $\mathrm{mD}$ (compare lanes 10 and 14). The residual ubiquitination of securin mDK was likely mediated by the TEK boxes that directly bound to UbcH10 (34). APC/C Cdc20 exhibited substantially different activities toward securin $\mathrm{mD}$ and $\mathrm{mDK}$ at multiple time points (Fig. $4 C$ ). These results suggested that, in the absence of its D box, the KEN box of securin became more critical for its ubiquitination by APC/C $\mathrm{Cdc}^{\mathrm{CdO}}$. Thus, the $\mathrm{KEN}$ box of securin can be a functional APC/C degron, but it makes little contribution to securin ubiquitination by APC/C $\mathrm{C}^{\mathrm{Cdc} 20}$ in wild-type securin.

The underlying mechanism for this observation was unclear, but it was conceivable that, in the wild type securin, the $\mathrm{D}$ box competed with the KEN box for ubiquitination by APC/C ${ }^{\mathrm{Cdc} 20}$. Consistent with this notion, a synthetic cyclin B1 D-box peptide inhibited the ubiquitination of securin $\mathrm{mD}$ (which was a KENbox substrate) (Fig. $4 B$, compare lanes 10 and 12). The D-box peptide also inhibited APC/C ${ }^{\mathrm{Cdc} 20}$-catalyzed ubiquitination of the securin $\Delta \mathrm{D}$ mutant with its $\mathrm{D}$ box deleted (Fig. $4 D$ ). These results suggested the D box might compete with the KEN box or other functional elements of securin for ubiquitination by APC/ $\mathrm{C}^{\mathrm{Cdc} 20}$, although we could not rule out the possibility that residues neighboring the $\mathrm{D}$ box assumed D-box-like functionality in the securin $\Delta \mathrm{D}$ mutant. Addition of the BubR1 KEN1 peptide at a concentration of $300 \mu \mathrm{M}$ did not reduce the ubiquitination of any forms of securin (Fig. 4B), consistent with the previous findings that additional regions of BubR1 or binding of Mad2 or both were required to inhibit $\operatorname{APC} / \mathrm{C}^{\operatorname{Cdc} 20}(16,25)$.

Low Cooperativity Between D and KEN Boxes in APC/C Cdh1 $^{\text {-Mediated }}$ Ubiquitination of Securin. We next examined the ubiquitination of various securin proteins by $\mathrm{APC} / \mathrm{C}^{\mathrm{Cdh} 1}$. Consistent with
A
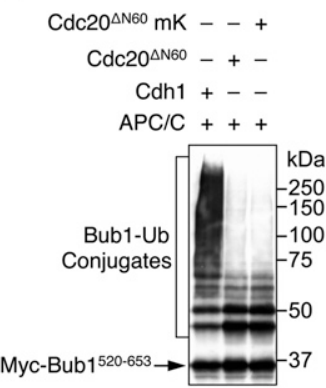

C

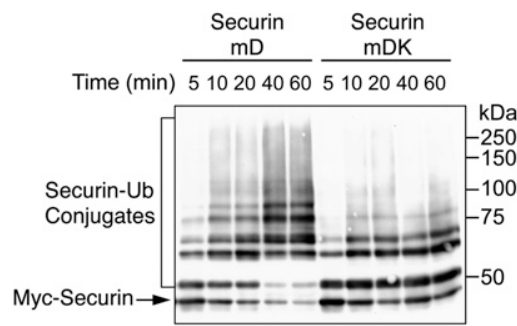

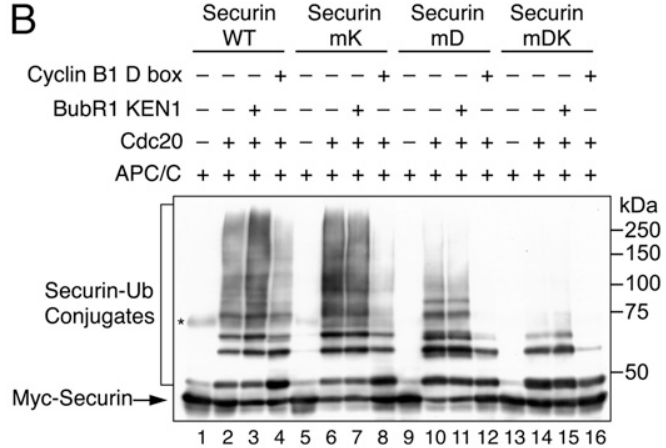

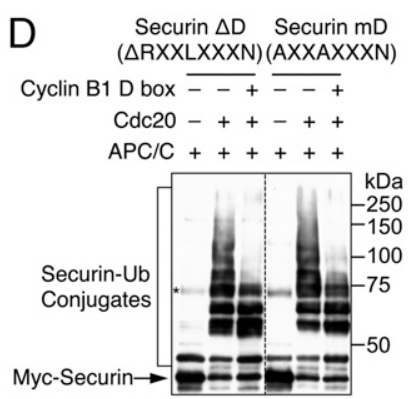

Fig. 4. The KEN box of securin supports $A P C / C^{C d c 20}$-dependent ubiquitination in the absence of the D box. $(A) C d h 1, C d c 20^{\triangle N 60}$ $\mathrm{WT}$, and $\mathrm{Cdc} 20^{\Delta \mathrm{N} 60} \mathrm{mK}$ were assayed for their ability to promote ubiquitination of Myc-Bub1 $1^{520-653}$ by APC/C. The reaction mixtures were blotted with anti-Myc. The positions of unmodified Bub1 and Bub1-ubiquitin conjugates are labeled. (B) Ubiquitination of Myc-securin WT, mK, mD, and mDK by APC/C ${ }^{\mathrm{Cd} c 20}$. The BubR1 KEN1 and cyclin B1 D-box peptide were added to $300 \mu \mathrm{M}$ and $150 \mu \mathrm{M}$, respectively, in the indicated lanes. The reaction time was $10 \mathrm{~min}$. The reaction mixtures were blotted with antiMyc. The positions of unmodified securin and securin-ubiquitin conjugates are labeled. A contaminating protein in the Mycsecurin preparations is indicated by an asterisk. (C) Ubiquitination of Myc-securin $\mathrm{mD}$ and $\mathrm{mDK}$ by APC/C $\mathrm{C}^{\mathrm{Cdc} 20}$ at the indicated times. The reaction mixtures were blotted with anti-Myc. The positions of unmodified securin and securin-ubiquitin conjugates are labeled. $(D)$ Ubiquitination of Myc-securin $\triangle D$ and $m D$ by APC/C ${ }^{\mathrm{Cdc} 20}$ for $2 \mathrm{~h}$. The cyclin B1 D-box peptide was added to $500 \mu \mathrm{M}$ in the indicated lanes. The reaction mixtures were blotted with anti-Myc. The positions of unmodified securin and securin-ubiquitin conjugates are labeled. A contaminating protein in the Myc-securin preparations is indicated by an asterisk. 
a previous report (28), mutation of either the KEN or D box reduced securin ubiquitination, whereas mutation of both largely abolished ubiquitination (Fig. 5A). In the presence of APC/ $\mathrm{C}^{\mathrm{Cdh} 1},{ }^{35} \mathrm{~S}$-securin WT formed higher molecular mass ubiquitin conjugates compared with ${ }^{35} \mathrm{~S}$-securin $\mathrm{mK}$ (Fig. $5 B$, compare lanes 3 and 6). Cold securin WT competed against ${ }^{35} \mathrm{~S}$-securin WT or $\mathrm{mK}$ for ubiquitination by $\mathrm{APC} / \mathrm{C}^{\mathrm{Cdh} 1}$ more effectively than cold securin $\mathrm{mK}$ did (Fig. $5 B$ ). These results indicated an involvement of the KEN box in APC/C ${ }^{\mathrm{Cdh} 1}$-dependent ubiquitination of securin. The fact that securin mutants with either box mutated still underwent considerable ubiquitination was inconsistent with highly cooperative binding of these two motifs to $\mathrm{APC} / \mathrm{C}^{\mathrm{Cdh} 1}$.

As expected, a synthetic securin KEN-box peptide blocked APC/C $C^{C d h 1}$-mediated ubiquitination of securin $\mathrm{mD}$, a KEN-box substrate (Fig. 5C, compare lanes 3 and 7). Unexpectedly, this KEN peptide also reduced the ubiquitination of securin $\mathrm{mK}$, a D-box substrate (Fig. 5C, compare lanes 2 and 6). Likewise, a synthetic securin D-box peptide not only blocked the ubiquitination of securin $\mathrm{mK}$ (compare lanes 2 and 10), but also reduced the ubiquitination of securin $\mathrm{mD}$ (compare lanes 3 and 11). These results could be explained by competition between the $\mathrm{D}$ and KEN boxes in APC/C Cdh1 -catalyzed ubiquitination, although we could not rule out the possibility that the mutated KEN and $\mathrm{D}$ boxes still had residual functionality.

To further confirm a competition between APC/C degrons, we tested the effects of several additional synthetic KEN-box and Dbox peptides on APC/C $\mathrm{C}^{\mathrm{Cdh} 1}$-dependent ubiquitination of securin $\mathrm{mD}$ and two other KEN-box substrates, Bub1 $1^{520-653}$ and the Nterminal domain of Cdc20. Three KEN-box peptides-Bub1 KEN1, BubR1 KEN1, and securin KEN-inhibited the ubiquitination of these substrates to varying degrees, with Bub1 KEN1 being the most effective (Fig. $5 D$ and $E$ ). Consistent with a competition between $\mathrm{D}$ and KEN boxes, the cyclin B1 and securin D-box peptides also inhibited the APC/C $C^{\mathrm{Cdh} 1}$-mediated ubiquitination of all three substrates. A cyclin B1 mutant D-box peptide with the RXXL motif mutated to AXXA did not inhibit Cdc20 ubiquitination (Fig. 5E), demonstrating the specificity of such a competition.

Low-Cooperativity Multisite Substrate Recognition by APC/C. Our structural analysis of human $\mathrm{Cdc} 20$, along with the recently published EM structures of APC/C and the crystal structure of the fission yeast MCC $(10,11,21)$, firmly establishes that the
APC/C activators, $\mathrm{Cdc} 20$ and $\mathrm{Cdh} 1$, use two distinct binding sites for binding the two key APC/C degrons: the $\mathrm{D}$ and $\mathrm{KEN}$ boxes. The KEN-box-binding site lies at the top face of the $\beta$ propeller, whereas a conserved patch located at the side of $\beta$ propeller, together with Apc10, forms the D-box-binding site.

Several APC/C substrates, including securin, contain both KEN and D boxes. Highly cooperative bivalent binding of both degrons of a given substrate to $\mathrm{APC} / \mathrm{C}$ is expected to yield a dissociation constant $\left(K_{\mathrm{d}}\right)$ between $\mathrm{APC} / \mathrm{C}$ and the substrate that is much smaller than either of the two individual $K_{\mathrm{d}} \mathrm{s}$ between $\mathrm{APC} / \mathrm{C}$ and each degron. In this scenario, mutation of either the KEN or D box greatly increases the $K_{\mathrm{m}}$ and impairs securin ubiquitination by APC/C. This does not appear to be the case in APC/C-catalyzed ubiquitination of securin. The KEN box contributes minimally to the ubiquitination of wild-type securin by $\mathrm{APC} / \mathrm{C}^{\mathrm{Cdc} 20}$, but becomes more critical for the ubiquitination of the D-box mutant of securin. Mutation of either box has small effects on securin ubiquitination by APC/C ${ }^{\mathrm{Cdh} 1}$. Furthermore, the D-box peptides decrease KEN-box-dependent substrate ubiquitination by APC/C. Taken together, our data are inconsistent with highly cooperative binding of the two degrons of securin to $\mathrm{APC} / \mathrm{C}$.

There is little cooperativity between the KEN and D boxes of securin in its ubiquitination by $\mathrm{APC} / \mathrm{C}^{\mathrm{Cdc} 20}$, whereas a weak cooperativity between the two motifs is observed in APC/C $\mathrm{C}^{\mathrm{Cdh} 1}$ dependent ubiquitination. The physical basis for the lack of strong cooperativity is unclear at present. The KEN and D boxes of securin are separated by more than 40 residues. This linker may be too long to optimally span the two degron-binding sites of Cdc20/Cdh1. The gain in binding energy by engaging both motifs may be neutralized by the loss in entropy. Indeed, the linkers between the KEN and D boxes of APC/C inhibitors, Acm 1 and Mes1, are substantially shorter and have only around 20 residues (35-37). Both the KEN and D boxes of Acm1 and Mes1 are required for their inhibition of APC/C (35-37). It is possible that the KEN and D boxes in these two proteins have higher cooperativity, allowing them to compete more effectively with bona fide APC/C substrates that have only one degron or have multiple degrons with little cooperativity.

We note that $A c m 1$ is not ubiquitinated by $\operatorname{APC} / C(35,36)$. Even more surprising, deletion of the KEN and D boxes increases its ubiquitination by $\mathrm{APC} / \mathrm{C}$, suggesting that the cooperative binding of KEN and D boxes seen in Acm 1 might actually hinder ubiquitination (36). Mes1 is both an inhibitor and a substrate of

A

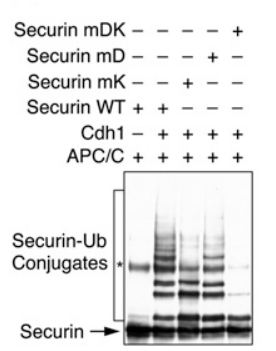

B

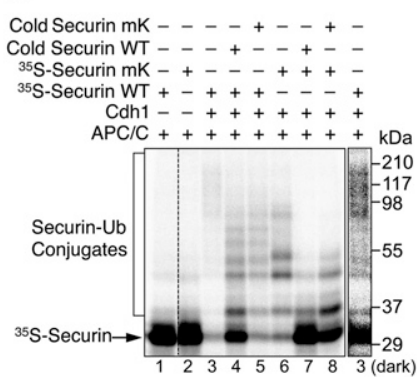

C

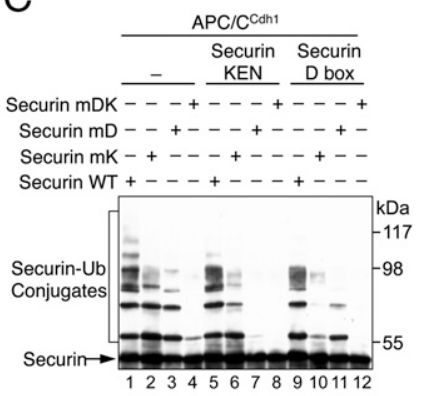

D

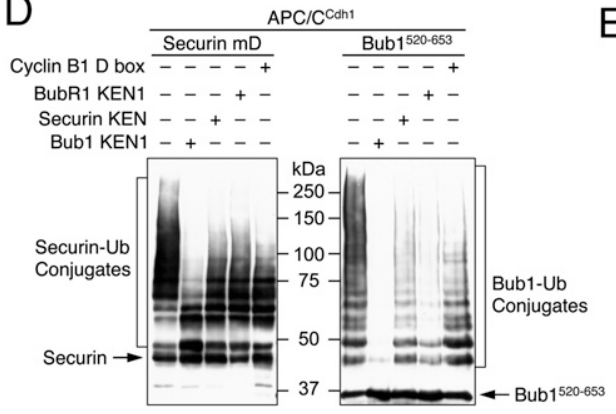

\section{$\mathrm{E}$}

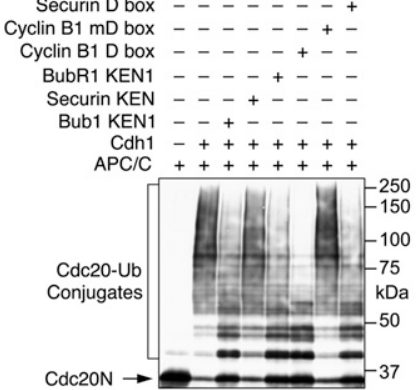

Fig. 5. Low cooperativity between the KEN and D boxes in $A P C / C^{C d h 1}$-mediated ubiquitination. $(A)$ Ubiquitination of Myc-securin $\mathrm{WT}, \mathrm{mK}, \mathrm{mD}$, and $\mathrm{mDK}$ by $A P C / C^{C d h} 1$ with $500 \mathrm{nM} \mathrm{UbCH10}$ for $20 \mathrm{~min}$. The reaction mixtures were blotted with anti-Myc. A contaminating protein in the Myc-securin preparations is indicated by an asterisk. (B) Ubiquitination of ${ }^{35} \mathrm{~S}$-labeled in vitro translated securin $\mathrm{WT}$ and $\mathrm{mK}$ by APC/ $C^{\text {Cdh1 }}$. Nonradioactive recombinant purified securin WT and $\mathrm{mK}$ were included in the indicated lanes as competitors. The reaction mixtures were resolved on SDS/PAGE and analyzed with a phosphoimager. (C) Ubiquitination of Myc-securin WT, $\mathrm{mK}, \mathrm{mD}$, and $\mathrm{mDK}$ by $\mathrm{APC} / \mathrm{C}^{\mathrm{Cdh} 1}$ with $500 \mathrm{nM} \mathrm{UbcH} 10$ for $20 \mathrm{~min}$. The securin $\mathrm{KEN}$ - and D-box peptides were added to $1 \mathrm{mM}$ in the indicated lanes. The reaction mixtures were blotted with anti-Myc. (D) Ubiquitination of Myc-securin mD (Left) and Bub1 ${ }^{520-653}$ (Right) by APC/C $C^{\text {Cdh1 }}$ for $1 \mathrm{~h}$. The Bub1 KEN1 (1 mM), securin KEN (1 mM), BubR1 KEN1 (307 $\mu \mathrm{M})$, and cyclin B1 Dbox $(153 \mu \mathrm{M})$ peptides were added to the indicated lanes. The reaction mixtures were blotted with antiMyc. $(E)$ Ubiquitination of in vitro translated Myctagged $\mathrm{N}$-terminal fragment of $\mathrm{Cdc} 20(\mathrm{Cdc} 20 \mathrm{~N})$ by $A P C / C^{C d h} 1$ for $2 \mathrm{~h}$. The indicated peptides were added at a final concentration of $150 \mu \mathrm{M}$. The reaction mixtures were blotted with anti-Myc. 
APC/C. As a substrate, the KEN and D boxes of Mes1 are not highly cooperative, as mutations of either motif do not impede its degradation in vivo and have little effects on its ubiquitination by $\mathrm{APC} / \mathrm{C}$ in vitro (37). So far, there are no known examples of $\mathrm{APC} / \mathrm{C}$ substrates exhibiting highly cooperative KEN- and D-boxdependent ubiquitination.

Our finding that D-box peptides inhibit the ubiquitination of KEN-box substrates in trans suggests a competition between the two motifs in the ubiquitination of certain APC/C substrates. The underlying mechanism of this competition is unclear at present. One possible explanation is that the engagement of the D box with Cdc20/Cdh1 and Apc10 disfavors KEN-box binding to $\mathrm{Cdc} 20 / \mathrm{Cdh} 1$, and, to a lesser degree, vice versa. On the other hand, we cannot rule out alternative explanations. For example, the D-box peptides could compete with yet unidentified D-boxlike functional elements within the APC/C substrates examined.

Regardless the mechanism, the lack of strong positive cooperativity in degron binding by APC/C means that each degron is capable of supporting substrate ubiquitination and degradation to some degree. This partial functional redundancy generally applies to the cellular degradation of APC/C substrates containing both the D and KEN boxes, including securin, Sgo1, and Mes1 (6, 28, $37,38)$. In these cases, mutation of both boxes is required to block substrate degradation. We propose that this functional redundancy ensures the robustness of APC/C-dependent substrate degradation and expands the repertoire of $\mathrm{APC} / \mathrm{C}$ substrates.

\section{Conclusion}

Our structural and biochemical analyses of the mitotic APC/C activator Cdc20 reveal low-cooperativity, multisite binding of APC/C

1. Peters JM (2006) The anaphase promoting complex/cyclosome: A machine designed to destroy. Nat Rev Mol Cell Biol 7(9):644-656.

2. Thornton BR, Toczyski DP (2006) Precise destruction: An emerging picture of the APC. Genes Dev 20(22):3069-3078.

3. Yu H (2007) Cdc20: A WD40 activator for a cell cycle degradation machine. Mol Cell 27 (1):3-16.

4. Glotzer M, Murray AW, Kirschner MW (1991) Cyclin is degraded by the ubiquitin pathway. Nature 349(6305):132-138.

5. Pfleger CM, Lee E, Kirschner MW (2001) Substrate recognition by the Cdc20 and Cdh1 components of the anaphase-promoting complex. Genes Dev 15(18):2396-2407.

6. Zur A, Brandeis M (2001) Securin degradation is mediated by fzy and fzr, and is required for complete chromatid separation but not for cytokinesis. EMBO J 20(4): 792-801.

7. Kraft C, Vodermaier HC, Maurer-Stroh S, Eisenhaber F, Peters JM (2005) The WD40 propeller domain of Cdh1 functions as a destruction box receptor for APC/C substrates. Mol Cell 18(5):543-553.

8. Carroll CW, Enquist-Newman M, Morgan DO (2005) The APC subunit Doc1 promotes recognition of the substrate destruction box. Curr Biol 15(1):11-18

9. Matyskiela ME, Morgan DO (2009) Analysis of activator-binding sites on the APC/C supports a cooperative substrate-binding mechanism. Mol Cell 34(1):68-80.

10. da Fonseca PC, et al. (2011) Structures of $A P C / C(C d h 1)$ with substrates identify $C d h 1$ and Apc10 as the D-box co-receptor. Nature 470(7333):274-278.

11. Buschhorn BA, et al. (2011) Substrate binding on the APC/C occurs between the coactivator Cdh1 and the processivity factor Doc1. Nat Struct Mol Biol 18(1):6-13.

12. Yu H (2002) Regulation of APC-Cdc20 by the spindle checkpoint. Curr Opin Cell Biol 14 (6):706-714.

13. Bharadwaj R, Yu H (2004) The spindle checkpoint, aneuploidy, and cancer. Oncogene 23(11):2016-2027.

14. Musacchio A, Salmon ED (2007) The spindle-assembly checkpoint in space and time. Nat Rev Mol Cell Biol 8(5):379-393.

15. Kim S, Yu H (2011) Mutual regulation between the spindle checkpoint and APC/C. Semin Cell Dev Biol 22(6):551-558.

16. Tang Z, Bharadwaj R, Li B, Yu H (2001) Mad2-Independent inhibition of APCCdc20 by the mitotic checkpoint protein BubR1. Dev Cell 1(2):227-237.

17. Fang G (2002) Checkpoint protein BubR1 acts synergistically with Mad2 to inhibit anaphase-promoting complex. Mol Biol Cell 13(3):755-766.

18. Sudakin V, Chan GK, Yen TJ (2001) Checkpoint inhibition of the APC/C in HeLa cells is mediated by a complex of BUBR1, BUB3, CDC20, and MAD2. J Cell Biol 154(5): 925-936.

19. Herzog F, et al. (2009) Structure of the anaphase-promoting complex/cyclosome interacting with a mitotic checkpoint complex. Science 323(5920):1477-1481.

20. Izawa D, Pines J (2011) How APC/C-Cdc20 changes its substrate specificity in mitosis. Nat Cell Biol 13(3):223-233.

21. Chao WC, Kulkarni K, Zhang Z, Kong EH, Barford D (2012) Structure of the mitotic checkpoint complex. Nature 484(7393):208-213.

22. Burton JL, Solomon MJ (2007) Mad3p, a pseudosubstrate inhibitor of APCCdc20 in the spindle assembly checkpoint. Genes Dev 21(6):655-667. degrons. This mode of substrate recognition has important implications for the mechanism and regulation of APC/C-dependent ubiquitination reactions. Chemical inhibitors of Cdc20 are expected to cause prolonged mitotic arrest and apoptosis in human cancer cells and thus have therapeutic potential. Our highresolution structure of human $\mathrm{Cdc} 20$ enables virtual screens of compound libraries for such inhibitors.

\section{Materials and Methods}

Protein Purification and Crystallization. Human Cdc20 proteins were expressed in insect cells and purified with affinity and conventional chromatography. Standard methodologies were used to crystallize Cdc20 alone or bound to BubR1 KEN1 and determine the structures. See SI Materials and Methods for details. Data collection and structure refinement statistics are summarized in Table S1.

Protein Binding and Ubiquitination Assays. Protein binding and ubiquitination assays were performed as described $(39,40)$. See SI Materials and Methods for details.

ACKNOWLEDGMENTS. We thank Dominika Borek for data processing, Xuewu Zhang for assistance with structure determination, Michael Rape for providing protein Ube2S, Haydn Ball for peptide synthesis, and Laura DiazMartinez for data analysis. Use of Argonne National Laboratory Structural Biology Center beamlines at the Advanced Photon Source was supported by the US Department of Energy under contract DE-AC02-06CH11357. This study is supported by National Institutes of Health Grant GM085004 (to X.L.), a Cancer Prevention Research Institute of Texas grant (to H.Y.), and Welch Foundation Grant I-1441 (to H.Y.). H.Y. is an Investigator with the Howard Hughes Medical Institute.

23. King EM, van der Sar SJ, Hardwick KG (2007) Mad3 KEN boxes mediate both Cdc20 and Mad3 turnover, and are critical for the spindle checkpoint. PLOS ONE 2(4): e342.

24. Sczaniecka M, et al. (2008) The spindle checkpoint functions of Mad3 and Mad2 depend on a Mad3 KEN box-mediated interaction with Cdc20-anaphase-promoting complex (APC/C). J Biol Chem 283(34):23039-23047.

25. Lara-Gonzalez P, Scott MI, Diez M, Sen O, Taylor SS (2011) BubR1 blocks substrate recruitment to the APC/C in a KEN-box-dependent manner. J Cell Sci 124(Pt 24): 4332-4345.

26. Han Z, et al. (2006) Structural basis for the specific recognition of methylated histone H3 lysine 4 by the WD-40 protein WDR5. Mol Cell 22(1):137-144.

27. Huang HC, Shi J, Orth JD, Mitchison TJ (2009) Evidence that mitotic exit is a better cancer therapeutic target than spindle assembly. Cancer Cell 16(4):347-358.

28. Hagting A, et al. (2002) Human securin proteolysis is controlled by the spindle checkpoint and reveals when the APC/C switches from activation by Cdc20 to Cdh1. J Cell Biol 157(7):1125-1137.

29. Wu T, et al. (2010) UBE2S drives elongation of K11-linked ubiquitin chains by the anaphase-promoting complex. Proc Natl Acad Sci USA 107(4):1355-1360.

30. Williamson A, et al. (2009) Identification of a physiological E2 module for the human anaphase-promoting complex. Proc Natl Acad Sci USA 106(43):18213-18218.

31. Garnett MJ, et al. (2009) UBE2S elongates ubiquitin chains on APC/C substrates to promote mitotic exit. Nat Cell Biol 11(11):1363-1369.

32. Qi W, Yu H (2007) KEN-box-dependent degradation of the Bub1 spindle checkpoint kinase by the anaphase-promoting complex/cyclosome. J Biol Chem 282(6): 3672-3679.

33. Kang J, et al. (2008) Structure and substrate recruitment of the human spindle checkpoint kinase Bub1. Mol Cell 32(3):394-405

34. Jin L, Williamson A, Banerjee S, Philipp I, Rape M (2008) Mechanism of ubiquitin-chain formation by the human anaphase-promoting complex. Cell 133(4):653-665.

35. Ostapenko D, Burton JL, Wang R, Solomon MJ (2008) Pseudosubstrate inhibition of the anaphase-promoting complex by Acm1: Regulation by proteolysis and $\mathrm{Cdc} 28$ phosphorylation. Mol Cell Biol 28(15):4653-4664.

36. Choi E, Dial JM, Jeong DE, Hall MC (2008) Unique D box and KEN box sequences limit ubiquitination of Acm 1 and promote pseudosubstrate inhibition of the anaphasepromoting complex. J Biol Chem 283(35):23701-23710.

37. Kimata $Y$, et al. (2008) A mutual inhibition between APC/C and its substrate Mes1 required for meiotic progression in fission yeast. Dev Cell 14(3):446-454.

38. Karamysheva Z, Diaz-Martinez LA, Crow SE, Li B, Yu H (2009) Multiple anaphasepromoting complex/cyclosome degrons mediate the degradation of human Sgo1. $J$ Biol Chem 284(3):1772-1780.

39. Tang Z, Yu H (2004) Functional analysis of the spindle-checkpoint proteins using an in vitro ubiquitination assay. Methods Mol Biol 281:227-242.

40. Tian W, Yu J, Tomchick DR, Pan D, Luo X (2010) Structural and functional analysis of the YAP-binding domain of human TEAD2. Proc Natl Acad Sci USA 107(16): 7293-7298. 